\title{
Messung von digitalem Stress im organisationalen Umfeld: Erfahrungen aus einer Fallstudie
}

\author{
Thomas Fischer (D) • René Riedl $\mathbb{D}$
}

Eingegangen: 2. September 2019 / Angenommen: 30. Januar 2020 / Online publiziert: 10. Februar 2020

(C) Der/die Autor(en) 2020

Zusammenfassung Digitaler Stress ist eine Form von Stress, die aus der Interaktion mit Informations- und Kommunikationstechnologien sowie deren Allgegenwärtigkeit in Wirtschaft und Gesellschaft resultiert. Digitaler Stress ist weit verbreitet und hat weitreichende negative Konsequenzen, wie etwa verringerte Zufriedenheit am Arbeitsplatz oder Burnout. Bislang ist die Erforschung von digitalem Stress vor allem auf Untersuchungen in Laborumgebungen sowie Online-Umfragen beschränkt. In diesem Artikel fokussieren wir auf einen anderen Forschungsansatz, nämlich Feldforschung. Es werden Methoden verglichen, die zur Erforschung von digitalem Stress am Arbeitsplatz eingesetzt werden können. Konkret wurden in einer Fallstudie über drei Arbeitswochen folgende Messinstrumente eingesetzt: Online-Fragebogen, Online-Tagebuch, Brustgurt zur Aufzeichnung der Herzschlagfrequenz sowie Gerät zur Messung von Blutdruck am Handgelenk. Insgesamt 16 Teilnehmer aus der Fallstudienorganisation nutzten diese Messinstrumente weitgehend selbständig, so dass Interventionen der beteiligten Forscher auf ein Mindestmaß reduziert werden konnten. Auf Basis der gewonnen Erkenntnisse werden die eingesetzten Methoden auf der Basis von sechs Gütekriterien (Reliabilität, Validität, Sensitivität, Diagnostizität, Objektivität, Aufdringlichkeit) verglichen. Es zeigt sich, dass jedes Messinstrument

Zusatzmaterial online Zusätzliche Informationen sind in der Online-Version dieses Artikels (https:// doi.org/10.1365/s40702-020-00596-w) enthalten.

T. Fischer $(\bowtie) \cdot$ R. Riedl

Fakultät für Wirtschaft \& Management, Abteilung für Digital Business, Fachhochschule

Oberösterreich, Wehrgrabengasse 1-3, 4400 Steyr, Österreich

E-Mail: thomas.fischer@fh-steyr.at

R. Riedl

E-Mail: rene.riedl@fh-steyr.at

R. Riedl

Institut für Wirtschaftsinformatik - Information Engineering, Johannes Kepler Universität Linz, Altenberger Straße 69, 4020 Linz, Österreich 
Stärken und Schwächen hat. Daraus folgt, dass zur praxisorientierten Erforschung von digitalem Stress ein Methoden-Mix angewendet werden sollte.

Schlüsselwörter Digitaler Stress · Feldstudie $\cdot$ Messmethodik · Befragung · Physiologie $\cdot$ NeuroIS

\title{
Measurement of Digital Stress in Organizations: Experiences from a Case Study
}

\begin{abstract}
Digital stress is a form of stress, which is caused by interaction with information and communication technologies and by their omnipresence in economy and society. This phenomenon is highly prevalent and it has substantial negative consequences such as reduced job satisfaction or burnout. So far, research on digital stress has been mostly confined to laboratory environments and online surveys. In this article, we focus on another research approach, namely field research. We compare methods that can be used to investigate digital stress in the workspace. Specifically, we conducted a case study over three workweeks using the following measurement methods: online surveys, online diary, a chest belt to measure heart rate and a device to measure blood pressure on the wrist. A total of 16 participants in the case organization used these measurement methods widely autonomously resulting in a minimal need for intervention by the researchers. Based on the experiences gathered through this case, the applied methods are compared using six criteria (i.e., reliability, validity, sensitivity, diagnosticity, objectivity, and intrusiveness). We find that each method has its strengths and weaknesses. Therefore, a mix of methods should be applied to practical research on digital stress.
\end{abstract}

Keywords Digital Stress · Field Study · Measurement · Survey · Physiology · NeuroIS

\section{Einleitung}

Digitaler Stress ist ein Phänomen, das durch die Interaktion mit Informations- und Kommunikationstechnologien (IKT) sowie durch deren Einfluss auf unseren Arbeitsalltag (z. B. voranschreitende Automatisierung) ausgelöst wird (Riedl 2013). Weiter zeigen Befunde empirischer Forschung, dass digitaler Stress zu einer Reihe von negativen Konsequenzen führen kann, unter anderem vermehrte physiologische Aktivierung wie der Ausschüttung von Stresshormonen (Riedl et al. 2012) sowie verringerte Leistungsfähigkeit (Tams et al. 2014).

Die Feststellung der Ausgeprägtheit von digitalem Stress ist für ein Unternehmen bedeutsam, um Bewältigungsmaßnahmen zu planen und umzusetzen. So kann durch regelmäßige Erhebungen beispielsweise überprüft werden, ob Interventionen wie Stressmanagement-Trainings sich positiv auf die Mitarbeiter auswirken und wie genau deren Wirkung aussieht (Arnetz 1996). Zudem ist es für Forscher wichtig, das Phänomen nicht nur in Laborumgebungen sowie in Online-Umfragen zu untersuchen, sondern auch in seinem natürlichen Kontext, also direkt im Unternehmen. 
Untersuchungen in einem konkreten Unternehmenskontext bringen jedoch häufig hohen Aufwand mit sich.

Faktoren wie die benötigte Zeit, um an den Ort der Untersuchung zu gelangen, die Zeit für die Erhebungen selbst (z.B. persönliche Interviews oder Beobachtungen) und der Koordinationsaufwand (z.B. zeitliche Planung von Interviews), machen dabei das Ideal einer Vollerhebung in einem Fallstudienunternehmen (Einbindung aller vom Phänomen betroffenen Personen, was im Regelfall die gesamte Belegschaft ist) oftmals unerreichbar.

In Anbetracht der hohen Bedeutung von praxisorientierter Forschung für den Erkenntnisfortschritt in der Wirtschaftsinformatik soll in diesem Artikel dargestellt werden, wie Stressmessungen im Unternehmenskontext ohne bedeutende Intervention durch die beteiligten Forscher durchgeführt werden können. Insbesondere werden vier Messinstrumente verglichen, um dadurch Aussagen zu deren Zweckmäßigkeit zu gewinnen. Die Erkenntnisse der Studie sollen helfen, Wissenschaftlern und Praktikern eine Entscheidungsgrundlage für die Auswahl eines Messinstrumentariums im Kontext digitaler Stress zu liefern.

\section{Die Fallstudie}

Von Dezember 2017 bis Juni 2018 wurde digitaler Stress in einem österreichischen Unternehmen untersucht. Es handelt sich dabei um ein Verlagshaus mit rund 25 Mitarbeitern am Firmenhauptsitz. Insgesamt 16 Mitarbeiter erklärten sich bereit, an der Studie teilzunehmen, wobei drei Messzeiträume (im März, Mai und Juni) zu jeweils einer Arbeitswoche (Montag bis Freitag) im Studienprotokoll definiert wurden.

Die Distanz von der Forschungseinrichtung zum Studienort (rund $120 \mathrm{~km}$ ) stellte eine Herausforderung für die beteiligten Forscher dar, da diese eine tägliche Anwesenheit am Studienort, beispielsweise für spontane Erhebungen (z. B. Interviews oder Beobachtungen), nur unter erheblichem zeitlichen Aufwand erlaubt hätte.

Vor diesem Hintergrund wurden vier Messinstrumente ausgewählt, die eine weitestgehend selbständige Messung durch die Studienteilnehmer erlauben und zudem bereits in der Forschung zum digitalen Stress erfolgreich eingesetzt wurden.

\section{Messinstrumente}

In Anlehnung an die bisherige Forschung zu digitalem Stress (siehe Fischer und Riedl 2017 für eine Übersicht der bislang eingesetzten Methoden und Instrumente) wurde eine Kombination aus Messinstrumenten zur Selbstauskunft durch die Teilnehmer (Fragebogen und Tagebuch) sowie zur Messung physiologischer Prozesse (Herzschlagrate und Blutdruck) gewählt.

Standardisierter Online-Fragebogen Am Ende jeder Arbeitswoche wurden die Teilnehmer zu einer Online-Befragung eingeladen, die mit dem Tool „SoSciSurvey“1

\footnotetext{
1 https://www.soscisurvey.de/.
} 
umgesetzt wurde. Während der ersten Erhebung dauerte das Ausfüllen des Fragebogens rund $45 \mathrm{~min}$, da auch Fragenkomplexe zur Person (z. B. Persönlichkeit und Einstellungen gegenüber Technologie) enthalten waren, bei den beiden weiteren Erhebungen dauerte das Ausfüllen im Durchschnitt rund $20 \mathrm{~min}$. Für den Großteil der Fragen wurde ein einheitliches Bewertungsschema in Form einer 7-stufigen Likert Skala (,1 - Stimme überhaupt nicht zu“ bis „,7 - Stimme völlig zu“) eingesetzt.

Tagebuch Um die täglichen Aktivitäten sowie die eingesetzten Technologien und mögliche Probleme mit diesen Technologien zu erfassen (in Anlehnung an die „Day Reconstruction Method“" von Kahneman et al. 2004), wurde ein Online-Tagebuch entwickelt (von der Forschungseinrichtung vergebene Auftragsentwicklung durch ein Softwareunternehmen). Das Tagebuch wurde in Microsoft Sharepoint ${ }^{\circledR}$ umgesetzt und war den Teilnehmern online zugänglich. Um den Aufwand für die Befüllung des Tagebuchs zu verringern, wurden im Vorfeld Interviews mit den Teilnehmern geführt und ein System mit Antwortmöglichkeiten konfiguriert.

Herzschlagrate (HR) Der Herzschlag und daraus abgeleitete Kennzahlen (z.B. Herzratenvariabilität, Baumgartner et al. 2018) können zeitnahe Auskunft über physiologische Aktivierung etwa in Folge von Stresswahrnehmungen geben. Mit dem Aufkommen von Geräten zur Messung physiologischer Indikatoren im privaten Umfeld (z.B. Sportuhren) wurde auch die Idee, solche Geräte für die Stressforschung zu nutzen, populär (z. B. Schellhammer et al. 2013). In der wissenschaftlichen Forschung werden solche Geräte zunehmend als Möglichkeit zur Datensammlung anerkannt, da sie insbesondere die Feldforschung vereinfachen (z. B. Fischer und Riedl 2018).

In der Studie wurden drei Technikkomponenten für die Messung der HR verwendet: (1) ein Polar H7 Brustgurt, der die physiologischen Signale sammelt und per Bluetooth an (2) ein iPhone SE übertragt, in dem diese mit (3) der mobilen App „Heart Rate Variability Logger“ verarbeitet werden (siehe auch Kalischko et al. 2019).

Blutdruck (BP) Ein weiterer physiologischer Indikator, der Auskunft über die Stressbelastung, insbesondere die chronische Belastung, geben kann, ist der Blutdruck und dessen Komponenten (systolischer und diastolischer Blutdruck, Fischer et al. 2017). In der Forschung zu digitalem Stress wurden BP-Messungen bereits mehrfach mit HR-Messungen kombiniert, weshalb diese Datenquelle als zusätzlicher Indikator ausgewählt wurde. Zudem gibt es in der BP-Messung Protokolle für Langzeitstudien, die als Basis für Stress-Studien genutzt werden können (mehr hierzu findet sich im Onlinematerial zu diesem Artikel).

In der vorliegenden Studie wurde mit einem Omron RS8 Gerät am Handgelenk gemessen. Nach einer kurzen Einschulung konnte die Messung von den Teilnehmern selbst durchgeführt werden, wobei vormittags (ca. eine halbe Stunde nach dem Eintreffen am Arbeitsplatz) und nachmittags (ca. eine halbe Stunde vor dem Verlassen des Arbeitsplatzes) jeweils zwei Mal gemessen wurde (um die Zuverlässigkeit der Werte sicherzustellen). 
Tab. 1 Gütekriterien für Messverfahren basierend auf Riedl et al. (2014), p. xxix

\begin{tabular}{|c|c|}
\hline Gütekriterium & Beschreibung \\
\hline Reliabilität & $\begin{array}{l}\text { „Ein [Messinstrument] ist dann reliabel (zuverlässig), wenn [es] das Merkmal, das [es] } \\
\text { misst, exakt, d.h. ohne Messfehler, misst“ (Moosbrugger und Kelava 2012, p. 11) }\end{array}$ \\
\hline Validität & $\begin{array}{l}\text { „Ein [Messinstrument] gilt dann als valide (,gültig“), wenn [es] das Merkmal, das [es] } \\
\text { messen soll, auch wirklich misst und nicht irgendein anderes“ (Moosbrugger und Kelava } \\
\text { 2012, p. 13) }\end{array}$ \\
\hline Sensitivität & $\begin{array}{l}\text { Unterschiedliche Ausprägungen eines Konstrukts können unterschieden werden (z. B. } \\
\text { wenig vs viel Stress). (nach Riedl et al. (2014)) }\end{array}$ \\
\hline Diagnostizität & $\begin{array}{l}\text { Das Konstrukt von Interesse kann von anderen Konstrukten abgegrenzt werden (z. B. } \\
\text { Stress von Emotion). (nach Riedl et al. (2014)) }\end{array}$ \\
\hline Objektivität & $\begin{array}{l}\text { Objektivität im Kontext von Stressoren bedeutet, dass die Messung (möglichst) unabhän- } \\
\text { gig von kognitiven und emotionalen Einflüssen auf individueller Ebene stattfindet (z. B. } \\
\text { ohne Angst vor den Folgen der Beantwortung von Fragen). (nach Frese und Zapf 1988) }\end{array}$ \\
\hline $\begin{array}{l}\text { Aufdringlich- } \\
\text { keit }\end{array}$ & $\begin{array}{l}\text { Die Messung beeinflusst nicht das Konstrukt von Interesse (z. B. die Messung selbst } \\
\text { erzeugt keinen Stress). (nach Riedl et al. (2014)) }\end{array}$ \\
\hline
\end{tabular}

\section{Evaluierung der Messinstrumente}

Für die vergleichende Bewertung der Instrumente werden hier die sechs Gütekriterien für Messverfahren von Riedl et al. (2014) herangezogen (siehe Tab. 1). Jedes dieser Gütekriterien wird nachfolgend für alle vier Messverfahren diskutiert, sowohl im Sinne einer generellen Bewertung als auch im Kontext der Studie und der hierbei gesammelten Erfahrungen.

\subsection{Reliabilität}

Im Fall des Fragebogens konnte durchgehend auf bestehende Instrumente zurückgegriffen werden, deren Reliabilität in verschiedenen Studien bestätigt wurde (z. B. Ragu-Nathan et al. 2008 zeigen in ihrer Studie mit Hilfe von zwei getrennten Datensätzen, dass die Anwendung ihres Fragebogens wiederholt zu ähnlichen Resultaten führt). Zudem wurde der Fragebogen mit einem Online-Tool mit einheitlichem Design und einheitlichen Interaktionsmöglichkeiten umgesetzt. Weitere Informationen zum Test und der regelmäßigen Überprüfung der Reliabilität von Fragebögen finden sich im Artikel von Straub und Gefen (2004).

Das Tagebuch wurde ebenfalls einheitlich gestaltet und Antwortmöglichkeiten zu den Aktivitäten waren vorgegeben. Die Antwortmöglichkeiten waren in vielen Fällen abstrahiert (z.B. „Qualitätskontrolle“), um über alle Teilnehmer hinweg anwendbar zu sein. Diese Abstraktion führte teilweise zu einem Verlust an Reliabilität, da die Antwortmöglichkeiten unterschiedliche Interpretationen zuließen und somit eine einheitliche Verwendung nicht vollständig sichergestellt werden konnte.

Die HR-Messung bietet laut Riedl et al. (2014) eine gute Reliabilität im Allgemeinen. Ein Setup mit mehreren Technikkomponenten wie in der vorliegenden Studie erhöht jedoch das Potenzial für Messfehler. So kam es etwa gelegentlich zu einem Verrutschen des Brustgurts oder zu Unterbrechungen der Bluetooth-Verbindung für die Datenübertragung kommen, was zu verzerrten Daten oder zu einem 
Datenverlust führte. Idealtypisch besteht daher eine hohe Reliabilität, jedoch nur, wenn störungsfrei gemessen werden kann.

Für die BP-Messung existiert eine Fülle möglicher Faktoren, welche die Messung beeinflussen können (z.B. Sitzposition, Ernährungsgewohnheiten, Messzeitpunkt, Fischer et al. 2017). Es ist daher insbesondere in Feldstudien, die nur eine geringe Form der Kontrolle über die Situation der Messung zulassen, schwierig, hohe Reliabilität zu erreichen. Die Teilnehmer wurden daher zu einer Auswahl an zu beachtenden Faktoren vorab geschult (z. B. bestimmte Sitzposition und Armposition). Es konnte trotzdem nicht in jedem Fall eine gültige Messung sichergestellt werden.

\subsection{Validität}

Es existieren spezifische Fragebogen-Instrumente, um digitalen Stress zu messen (z.B. „Technostress Creators“ von Ragu-Nathan et al. 2008). Da sich die Quellen von digitalem Stress (z. B. Arten von Technologien) jedoch ändern, muss die Validität solcher standardisierter Instrumente regelmäßig geprüft werden (z. B. durch Befragungen in denen die Bestandteile eines Instruments auf ihre Relevanz und Vollständigkeit überprüft werden wie in der Studie von Fischer et al. 2019). Weitere Informationen zum Test und der regelmäßigen Überprüfung der Validität von Fragebögen finden sich im Artikel von Straub und Gefen (2004).

Das Tagebuch erlaubt hierbei mehr Spielraum, da auch weitere Technologien sowie Freitext für die Beschreibung von Problemen im Umgang mit Technologien hinzugefügt werden konnten. Anzumerken ist hierbei, dass in der Regel nur Problemfälle berichtet werden, die aus Sicht der Teilnehmer bereits ein höheres Niveau an Stress verursacht haben (d.h. alltägliche Probleme werden kaum berichtet). Dies führt in weiterer Folge vor allem zu einer Einschränkung der Sensitivität dieses Messverfahrens.

Für die physiologischen Messverfahren weisen Riedl et al. (2014) darauf hin, dass es üblicherweise aus Sicht der Wirtschaftsinformatik unzweckmäßig ist, einen physiologischen Indikator isoliert zu betrachten, da dieser im Regelfall mit zu vielen theoretischen Konstrukten in Verbindung stehen kann. Dies zeigte sich in dieser Studie vor allem für BP, da dieser über den Tagesverlauf von vielen möglichen Stressoren beeinflusst werden kann und eine Isolierung von digitalem Stress nur schwer möglich ist. Der Umstand, dass die HR-Messung kontinuierlich ist (und nicht wie die BP-Messung zu diskreten Zeitpunkten erfolgt), verleiht den Messwerten eine höhere Aussagekraft. Dennoch gilt, dass die Validität einer physiologischen Messung von digitalem Stress ohne zusätzliche Datenquellen zur Triangulation (z. B. Berichte aus dem Tagebuch) in einer Feldstudie eingeschränkt ist.

\subsection{Sensitivität}

Im Fall des Fragebogens wurde mehrheitlich mit einer Skala von 1 (niedrige Ausprägung) bis 7 (hohe Ausprägung) gemessen, wobei zudem jedes Konstrukt mit mehreren Fragestellungen gemessen wurde, um die Reliabilität, Validität und Sensitivität zu erhöhen (z.B. anstatt einer globalen Frage „Empfinden Sie digitalen Stress?" wird etwa nach einzelnen Quellen für digitalen Stress wie Überlastung 
durch zu viele E-Mails oder zu hohe Komplexität der eingesetzten Technologien gefragt).

Bezüglich Tagebuch stellten wir fest, dass das Vermerken von Vorfällen oftmals nur bei als außergewöhnlich wahrgenommenen Stressfällen geschieht (z. B. Absturz eines dringend benötigten Programms). Dieser Umstand schränkt die Sensitivität des Instruments ein. Es war zudem für die Teilnehmer schwierig, ihren Arbeitsalltag in ausreichender Granularität vollständig abzubilden, da oft zwischen Aufgaben innerhalb von Minuten hin und her gewechselt wird.

Mit einem Datenpunkt pro Sekunde weist die HR-Messung die mit Abstand größte Granularität unter den genutzten Verfahren auf. Zudem passt sich die Herzschlagrate in kürzester Zeit an Stresssituationen an (wenige Sekunden, z. B. Kalischko et al. 2019).

Aufgrund des Aufwands für die Studienteilnehmer, der mit wiederholten BPMessungen während des Arbeitstages verbunden ist, wurde nur zu zwei Zeitpunkten pro Arbeitstag gemessen, um darauf aufbauend den Tagesdurchschnitt zu berechnen. BP weist damit lediglich eine höhere Granularität als die Fragebogen-Messung auf.

\subsection{Diagnostizität}

Im Fall des Fragebogens werden mehrere Fragestellungen genutzt, um Auskunft über ein Konstrukt zu erhalten (z. B. 23 Fragestellungen für digitalen Stress im Messinstrument von Ragu-Nathan et al. 2008). Die einzelnen Fragestellungen (Items) können dabei vielen möglichen Konstrukten zugeordnet werden und sind nur im Zusammenspiel für ein bestimmtes Konstrukt aussagekräftig. Die Diagnostizität wird dabei in wiederholten Studien mit anderen Konstrukten nachgewiesen (z.B. indem statistische Verfahren zur Überprüfung auf diskriminante Validität angewendet werden).

Das Tagebuch erlaubt ebenfalls eine spezifische Einschränkung auf Stress, der durch IKT ausgelöst wird, wobei im Fall des genutzten Instruments eine gesamte Situation, die Stress ausgelöst hat, erfasst wird. Dadurch können Verflechtungen von Stressoren (z. B. Probleme mit einem System bei zeitgleichem Druck durch einen Abgabetermin) nicht ausgeschlossen werden.

Die physiologischen Indikatoren $(H R$ und $B P$ ) erlauben deutlich mehr Interpretationsspielraum und ein direkter Rückschluss auf digitalen Stress ist daher selten möglich (Riedl et al. 2014). Im Sinne der Diagnostizität, wie auch bei der Validität, ist eine Kombination mit anderen Verfahren empfehlenswert.

\subsection{Objektivität}

Eine bewusste Angabe unrichtiger Daten beim Fragebogen kann kaum ausgeschlossen werden. In der vorliegenden Studie ist das insofern relevant, da Fragestellungen oft eine Rückführung auf das zu messende Konstrukt zulassen. Sollten daher seitens Studienteilnehmern Bedenken bezüglich einer wahrheitsgetreuen Beantwortung existieren (z. B. im Fall einer Feldstudie, wenn die Anzahl der Teilnehmer beschränkt ist und somit Zweifel in Bezug auf die Anonymität bestehen, da demographische Daten wie Geschlecht und Alter erfasst werden), dann kann eine subjektive Beein- 
flussung nicht ausgeschlossen werden. Man denke beispielsweise daran, dass ein Teilnehmer statt dem Maximalwert einen geringeren Wert auswählt, um gegenüber den Forschern weniger gestresst zu wirken.

Auch im Fall des Tagebuchs, das noch direkter auf Einzelpersonen rückführbar ist (z. B. über die spezifischen Tätigkeiten), können Falschangaben kaum ausgeschlossen werden (z.B. kein Reporting, wenn etwa Angst besteht, dass man damit gegenüber dem Management negativ auffällig werden könnte).

Die physiologischen Indikatoren und insbesondere die HR-Messung sind für derartige Manipulationen weniger anfällig. Die HR-Messung erfolgt kontinuierlich und ist laut den Berichten der Teilnehmer rasch aus deren Aufmerksamkeit verschwunden. Bei der BP-Messung hingegen ist eine Manipulation leichter möglich, da nur geringe Veränderungen (z. B. Armposition) dazu führen, dass die gemessenen Werte höher oder niedriger sind.

\subsection{Aufdringlichkeit}

Das Ausfüllen des Fragebogens bedeutete für die Teilnehmer im Durchschnitt rund 20 bis 30 min Aufwand pro Arbeitswoche. Bei der ersten Erhebung gab es 15 vollständig ausgefüllt Fragebögen, bei der zweiten Erhebung 10 und bei der dritten Erhebung 8. Insgesamt gab es 5 Teilnehmer, die jeden der drei Fragebögen vollständig ausgefüllt haben (ausgehend von 16 Studienteilnehmern zu Beginn).

Beim Tagebuch wurde bereits ein Arbeitstag, an dem zumindest ein Eintrag gemacht wurde, als ,erfolgreich“ angesehen und es dauerte im Durchschnitt 20 bis 30 min pro Arbeitstag, um es zu befüllen (bei Einträgen, die eine Granularität von 30-min-Aktivitäten haben). Während der ersten Erhebung gab es 57 Tage mit Einträgen $(71 \%$ von max. $80 ; 80=16$ Personen $\times 5$ Arbeitstage), während der zweiten Erhebung 45 (56\% von 80) und während der dritten Erhebung 37 Tage (46\% von 80). Qualitativ gab es hier zudem auch Einbußen, da nur eine kleine Gruppe der Teilnehmer das Tagebuch im selben Detailgrad über alle drei Arbeitswochen hinweg befüllte.

Im Idealfall beträgt der zeitliche Aufwand für die HR-Messung nur rund 5 min pro Tag, dieser kann sich jedoch erhöhen, wenn etwa die Bluetooth-Verbindung zwischen Brustgurt und Smartphone unterbrochen wird (z. B. wenn das Smartphone am Arbeitsplatz vergessen wird und sich der Teilnehmer zu weit davon wegbewegt). Während der ersten Erhebung konnten 47 verwertbare Datensätze gesammelt werden (59\% von 80 ), während der zweiten Erhebung 36 (45\% von 80 ) und während der dritten Erhebung 44 (55\% von 80).

Die BP-Messung bedeutete für die Teilnehmer rund $10 \mathrm{~min}$ an zeitlichem Aufwand pro Tag (rund 5 min pro Messung). Während der ersten Erhebung konnten 76 verwertbare Datensätze gesammelt werden (48\% von 160;160=16 Teilnehmer $\times 5$ Arbeitstage $\times 2$ Messungen pro Tag), während der zweiten Erhebung 59 (37\% von 160) und während der dritten Erhebung 40 (25\% von 160). 
Tab. 2 Vergleichendes Ranking der Messverfahren

\begin{tabular}{lllll}
\hline & Fragebogen & Tagebuch & HR & BP \\
\hline Reliabilität & 1 & 3 & 2 & 4 \\
Validität & 1 & 2 & 3 & 4 \\
Sensitivität & 3 & 4 & 1 & 2 \\
Diagnostizität & 1 & 2 & 3 & 4 \\
Objektivität & 2 & 4 & 1 & 3 \\
Aufdringlichkeit & 2 & 3 & 1 & 4 \\
\hline
\end{tabular}

\section{5 Übergreifende Bewertung}

Auf Basis der Argumentation im vorangegangenen Abschnitt wurde in Tab. 2 ein Ranking der Messverfahren in Bezug auf jedes der sechs Gütekriterien vorgenommen. In dieses Ranking ( $1=$ beste Platzierung) fließen sowohl die allgemeinen Eigenschaften der Messverfahren nach Riedl et al. (2014) ein als auch die Erfahrungen aus der vorgestellten Fallstudie.

Es sollte dabei beachtet werden, dass es sich um ein relatives Ranking handelt, welches aus den subjektiven Eindrücken der Autoren hervorgeht. Zudem handelt es sich um eine Bewertung für einen spezifischen Untersuchungskontext, nämlich die Messung von digitalem Stress in einer Langzeitstudie im organisationalen Umfeld ohne direkte Intervention durch die beteiligten Forscher. Trotz des speziellen Untersuchungsgegenstands lassen sich Empfehlungen ableiten, vor allem was den Einsatzbereich der einzelnen Instrumente betrifft.

So eignet sich der Fragebogen, sofern ein spezifisches Fragebogen-Instrument in der Fachliteratur existiert, dazu, Wahrnehmungen der Teilnehmer zu erfassen und um mit wenig Aufwand ein Stimmungsbild der Situation im Unternehmen zu erhalten (z.B. aktuelles Ausmaß an digitalem Stress im gesamten Unternehmen). Je standardisierter dabei das Vorgehen (z. B. ein einheitliches Set an Fragestellungen), desto geringer der Aufwand für die Teilnehmer und die Forscher (z.B. bei der Datenauswertung). Standardisierung bedeutet jedoch auch, dass nötige Details verloren gehen (z. B. Welche Systeme lösen Stress bei den Teilnehmern aus? Welche Nutzungsszenarien sind verbesserungswürdig?).

Derartige Details können mit Hilfe eines Tagebuchs gesammelt werden (z.B. tägliche Probleme im Umgang mit verwendeten Technologien). Ein zu hoher Detaillierungsgrad ist beim Tagebuch jedoch nicht zweckdienlich, da dies einen inakzeptablen Aufwand für die Studienteilnehmer bedeuten würde (z. B. eine Notiz für jede Situation, in der auch nur ein geringes Maß an Stress mit einer Funktion einer Software empfunden wird).

Zusätzlich zur subjektiven Wahrnehmung und Bewertung durch die Teilnehmer erlaubt die HR-Messung eine Bestimmung der physiologischen Aktivierung der Teilnehmer in Echtzeit. Stresssituationen, die als ,alltäglich“ wahrgenommen werden, oder weitere Belastungen, die in einer subjektiven Bewertung womöglich untergehen (z. B. weil Probleme im Umgang mit IKT aus Scham oder Angst vor Jobverlust nicht berichtet werden), können mit diesem Verfahren identifiziert werden. Da physiologische Aktivierung jedoch viele Gründe haben kann (z. B. Bewegung am Arbeitsplatz 
oder emotionale Erregung abseits von digitalem Stress), sollte diese kontinuierliche Messung ein Ausgangspunkt für weitere, spezifischere Erhebungen sein (z.B. Abgleich mit dem Tagebuch oder Interviews mit den Teilnehmern).

Die BP-Messung lieferte in dieser Studie nur wenig zusätzliche Information über die Daten der anderen Messverfahren hinaus. Da es sich jedoch um ein Verfahren handelt, mit der viele Teilnehmer vertraut sind, kann sie als Erweiterung des Methodensets dienen, insbesondere da erhöhter Blutdruck eine mögliche Langzeitfolge von chronischem Stress ist.

\section{Zusammenfassung}

Im Zuge einer Fallstudie wurden vier Messverfahren (Fragebogen, Tagebuch, HRMessung, BP-Messung) zur Untersuchung von digitalem Stress im Unternehmensumfeld verglichen. Es zeigte sich, dass die Methoden unterschiedliche Aspekte des Phänomens erschließen und sich ihre jeweiligen Stärken und Schwächen ergänzen. Neben der Erkenntnis, dass ein komplementärer Einsatz verschiedener Verfahren für die Erfassung von digitalem Stress unerlässlich ist, hat sich auch gezeigt, dass Messgeräte aus dem privaten Umfeld (z.B. Brustgurte zur HR-Messung) für die Feldforschung in diesem Bereich geeignet sind (vgl. dazu auch Fischer und Riedl 2018). Dieser Ansatz sollte weiterverfolgt werden (z. B. mit Geräten, die mehrere Sensoren kombinieren), wobei auf die wissenschaftliche Güte der Instrumente geachtet werden muss (z.B. Reliabilität und Validität). Für die Praxis sind die Ergebnisse dieser Studie von Interesse, da die genutzten Messinstrumente regelmäßig eingesetzt werden können, ohne für die Beteiligten unnötig hohen Aufwand zu verursachen. Damit wird das Folgende erreicht: (i) Diagnose von digitalem Stress im Unternehmen (z.B. durch eine erste Erhebung mit Fragebögen), (ii) Identifikation von Stressursachen (z. B. mit Tagebüchern und begleitenden Interviews), (iii) Ableitung von Bewältigungsmaßnahmen (durch Analyse der Ergebnisse aus i und ii) sowie (iv) Überprüfung der Wirksamkeit der Maßnahmen (z.B. durch erneute Befragungen und kontinuierliche physiologische Messungen). Im Onlinematerial zu diesem Artikel werden die Messinstrumente weiter konkretisiert, wodurch die Planung und Durchführung ähnlicher Erhebungen in Unternehmen erleichtert wird.

Zudem stellt diese Studie nicht nur eine Form der Vorarbeit dar, um Forschung in der Praxis zu ermöglichen (z. B. indem die vorgestellten Methoden von Unternehmen eingesetzt werden), sondern auch um Forschung für die Praxis zu ermöglichen. Beispielsweise können die im Unternehmensumfeld erhobenen Daten dazu genutzt werden, in weiterführender Forschung Software zu entwickeln, die dabei hilft, Stress zu vermeiden (z. B. in Form von Stress-sensitiven Systemen, die im Fall von Stress die Arbeitsbelastung verringern, Adam et al. 2017), oder um Interventionen zu gestalten, die auf die jeweiligen Stressursachen im Unternehmen abgestimmt sind (z.B. wenn höhere Autonomie bei der Arbeit ermöglicht wird, damit flexibel mit den Anforderungen von IKT am Arbeitsplatz umgegangen werden kann, wie etwa Unterbrechungen durch E-Mails oder Systemnachrichten, Galluch et al. 2015). Weitere Beispiele und Denkanstöße zu solchen Formen der Forschung für die Praxis 
auf Basis von Messmethoden, die Feldforschung wie in dieser Studie aufgezeigt, erlauben, finden sich ebenso im Onlinematerial zu diesem Artikel.

Funding Open access funding provided by University of Applied Sciences Upper Austria.

Open Access Dieser Artikel wird unter der Creative Commons Namensnennung 4.0 International Lizenz veröffentlicht, welche die Nutzung, Vervielfältigung, Bearbeitung, Verbreitung und Wiedergabe in jeglichem Medium und Format erlaubt, sofern Sie den/die ursprünglichen Autor(en) und die Quelle ordnungsgemäß nennen, einen Link zur Creative Commons Lizenz beifügen und angeben, ob Änderungen vorgenommen wurden.

Die in diesem Artikel enthaltenen Bilder und sonstiges Drittmaterial unterliegen ebenfalls der genannten Creative Commons Lizenz, sofern sich aus der Abbildungslegende nichts anderes ergibt. Sofern das betreffende Material nicht unter der genannten Creative Commons Lizenz steht und die betreffende Handlung nicht nach gesetzlichen Vorschriften erlaubt ist, ist für die oben aufgeführten Weiterverwendungen des Materials die Einwilligung des jeweiligen Rechteinhabers einzuholen.

Weitere Details zur Lizenz entnehmen Sie bitte der Lizenzinformation auf http://creativecommons.org/ licenses/by/4.0/deed.de.

\section{Literatur}

Adam MTP, Gimpel H, Maedche A, Riedl R (2017) Design blueprint for stress-sensitive adaptive enterprise systems. Bus Inf Syst Eng 59:277-291. https://doi.org/10.1007/s12599-016-0451-3

Arnetz BB (1996) Techno-stress: a prospective psychophysiological study of the impact of a controlled stress-reduction program in advanced telecommunication systems design work. J Occup Environ Med 38:53-65

Baumgartner D, Fischer T, Riedl R, Dreiseitl S (2018) Analysis of heart rate variability (HRV) feature robustness for measuring technostress. In: Davis FD, Riedl R, Vom Brocke J, Léger P-M, Randolph A (Hrsg) Information systems and neuroscience: NeuroIS retreat 2018. Springer, Berlin Heidelberg, S 221-228

Fischer T, Riedl R (2017) Technostress research: a nurturing ground for measurement pluralism? Commun Assoc Inf Syst 40:375-401

Fischer T, Riedl R (2018) Lifelogging for organizational stress measurement; theory and applications. Springer, Cham, Switzerland

Fischer T, Halmerbauer G, Meyr E, Riedl R (2017) Blood pressure measurement: a classic of stress measurement and its role in technostress research. In: Davis FD, Riedl R, Vom Brocke J, Léger P-M, Randolph AB (Hrsg) Information systems and neuroscience. Gmunden retreat on NeuroIS 2017. Springer, Cham, S 25-35

Fischer T, Pehböck A, Riedl R (2019) Is the Technostress creators inventory still an up-to-date measurement instrument? Results of a large-scale interview study. In: Ludwig T, Pipek V (Hrsg) Proceedings of the 14. International Conference on Wirtschaftsinformatik, Siegen, Deutschland; 23.-27. Februar 2019, S 1834-1845

Frese M, Zapf D (1988) Methodological issues in the study of work stress: objective vs subjective measurement of work stress and the question of longitudinal studies. In: Cooper CL (Hrsg) Causes, coping and consequences of stress at work. Wiley, Chichester, S 375-411

Galluch P, Grover V, Thatcher JB (2015) Interrupting the workplace: examining stressors in an information technology context. J Assoc Inf Syst 16:1-47

Kahneman D, Krueger AB, Schkade DA, Schwarz N, Stone AA (2004) A survey method for characterizing daily life experience: the day reconstruction method. Science 306:1776-1780. https://doi.org/ 10.1126/science. 1103572

Kalischko T, Fischer T, Riedl R (2019) Techno-unreliability: a pilot study in the field. In: Davis FD, Riedl R, Vom Brocke J, Léger P-M, Randolph A (Hrsg) Information systems and neuroscience: NeuroIS retreat 2019. Springer, Berlin, Heidelberg, S 137-145

Moosbrugger H, Kelava A (2012) Qualitätsanforderungan an einen psychologischen Test (Testgütekriterien). In: Moosbrugger H, Kelava A (Hrsg) Testtheorie und Fragebogenkonstruktion, S 7-26 
Ragu-Nathan TS, Tarafdar M, Ragu-Nathan BS, Tu Q (2008) The consequences of technostress for end users in organizations: conceptual development and empirical validation. Information Systems Research 19:417-433

Riedl R (2013) On the biology of technostress: literature review and research agenda. Data Base Adv Inf Syst 44:18-55

Riedl R, Davis FD, Hevner AR (2014) Towards a NeuroIS research methodology: intensifying the discussion on methods, tools, and measurement. J Assoc Inf Syst i(15):i-xxxv

Riedl R, Kindermann H, Auinger A, Javor A (2012) Technostress from a Neurobiological perspective system breakdown increases the stress hormone cortisol in computer users. Bus Inf Syst Eng 4:61-69

Schellhammer S, Haines R, Klein S (2013) Investigating technostress in situ: understanding the day and the life of a knowledge worker using heart rate variability. In: IEEE (Hrsg) Proceedings of HICSS 2013, Wailea, HI, USA; 7.-10. Jänner 2013, S 430-439

Straub D, Gefen D (2004) Validation guidelines for IS Positivist research. CAIS. https://doi.org/10.17705/ 1CAIS.01324

Tams S, Hill K, Ortiz de Guinea A, Thatcher J, Grover V (2014) NeuroIS - alternative or complement to existing methods? Illustrating the holistic effects of neuroscience and self-reported data in the context of technostress research. J Assoc Inf Syst 15:723-753 